Audiology

Neurotology
Audiol Neurotol 2012;17:386-394

DOI: $\underline{10.1159 / 000341985}$
Received: May 21, 2012

Accepted: July 19, 2012

Published online: August 28, 2012

\title{
Can Long-Term Hearing Preservation be Expected in Children following Cholesteatoma Surgery? Results from a 14-Year-Long Study of Atticotomy-Limited Mastoidectomy with Cartilage Reconstruction
}

\author{
Chin-Lung Kuo An-Suey Shiao Wen-Huei Liao Ching-Yin Ho \\ Chiang-Feng Lien \\ Department of Otorhinolaryngology - Head and Neck Surgery, Taipei Veterans General Hospital and \\ National Yang-Ming University School of Medicine, Taipei, Taiwan, ROC
}

\section{Key Words}

Child • Cholesteatoma - Mastoid • Treatment outcome •

Tympanoplasty

\begin{abstract}
Objective: Following cholesteatoma surgery, effective longterm hearing preservation in children is difficult and is not typically expected. Hence, long-term data on hearing outcomes are lacking. The aim of this study was to analyze longterm hearing outcomes in children following cholesteatoma surgery. Methods: For this study, 49 ears in 47 children ( $\leq 16$ years) with acquired cholesteatomas following atticotomylimited mastoidectomy with cartilage reconstruction (insideout approach) during 1986-2010 were included. Pre- and post-operative recidivism-free audiometric results were compared. Hearing success was defined as a post-operative air conduction (AC) threshold of $\leq 30 \mathrm{~dB}$ (serviceable hearing). Logistic regression analyses were used to evaluate potential prognostic factors that independently contributed to the prediction of hearing success. These factors included stapes condition, pre-operative AC threshold, ossicular chain integrity, disease severity, age, and gender. Results: The mean
\end{abstract}

duration of follow-up was 14.2 years. The post-operative AC $(33.55 \pm 15.42 \mathrm{~dB})$ and air-bone gap $(17.88 \pm 12.94 \mathrm{~dB})$ were significantly improved compared with the pre-operative $A C$ $(42.90 \pm 16.47 \mathrm{~dB}, \mathrm{p}<0.001)$ and air-bone gap $(30.23 \pm 13.68$ $d B, p<0.001)$. The probability of hearing success following surgery $(40.8 \%)$ was significantly higher than prior to surgery (24.5\%, $p=0.008)$. Multivariate logistic regression analyses revealed a statistically significant correlation between hearing success and stapes integrity only $(p=0.005)$. Conclusions: This study provides important information on effective long-term hearing preservation over a mean follow-up of 14 years. In addition, stapes destruction is an independent negative prognostic determinant of achieving hearing success. The prediction model in this study provides otologists with useful pre-operative information to inform patients and parents on expected hearing outcomes and may be useful for post-operative observations.

Copyright $\odot 2012$ S. Karger AG, Basel

This work was presented in part as an invited lecture at the 11th Japan-Taiwan Conference on Otolaryngology - Head and Neck Surgery, Kobe, Japan, December 8-9, 2011.

\section{KARGER \\ Fax +4161306 1234 \\ E-Mail karger@karger.ch}

www.karger.com (c) 2012 S. Karger AG, Basel

$1420-3030 / 12 / 0176-0386 \$ 38.00 / 0$

Accessible online at:

www.karger.com/aud
Dr. Chiang-Feng Lien

Department of Otorhinolaryngology - Head and Neck Surgery

Taipei Veterans General Hospital

201, Section 2, Shih-Pai Road, Taipei 112, Taiwan (ROC)

Tel. +88622875 7763, E-Mail cflien@vghtpe.gov.tw; drtgud@gmail.com 
Table 1. CAO staging system of cholesteatoma

\begin{tabular}{llll}
\hline Score & C (cholesteatoma extent) & A (atelectatic eardrum) & O (ossicle destruction) \\
\hline 1 & 1 area of middle ear cleft & normal or grade I atelectasis & normal or destruction of 1 ossicle \\
2 & 2 areas of middle ear cleft & grade II or III atelectasis & $\begin{array}{l}\text { destruction of 2 ossicles } \\
\text { destruction of 3 ossicles }\end{array}$ \\
\hline
\end{tabular}

${ }^{a}$ The five areas of the middle ear cleft are tympanic cavity, attic, aditus, antrum, and mastoid cavity.

${ }^{\mathrm{b}}$ Degree of atelectasis according to Sadé [Sadé and Halevy, 1976] and Tos [Tos and Poulsen, 1980] classifications. In cases of eardrum perforation, destruction of $<50 \%$ of the middle ear mucosa is scored as 2 and $>50 \%$ is scored as 3 .

\section{Introduction}

Hearing impairment may cause deficits in a child's communication skills, which may result in learning problems and reduced academic achievement if the proper diagnosis and management are not applied [Most, 2004]. These are the reasons why hearing impairment in childhood is completely different from hearing loss in adulthood. In children with cholesteatoma, otologists should pay particular attention to post-operative hearing outcomes. However, functional hearing preservation is not typically expected and has rarely been an issue of concern because the surgeon's priority is to correct the disease, thus leading to an overemphasis on surgical outcomes (e.g. recurrence rate) [Hamilton, 2010]. Although a few investigations have been published that address the postoperative hearing outcomes of pediatric cholesteatoma surgeries, these studies lack sufficient follow-up to enable conclusions on long-term outcomes [Murphy and Wallis, 1998; Iino et al., 2001; Sun et al., 2010]. The aim of this study was to evaluate long-term hearing outcomes in children following cholesteatoma surgery.

\section{Materials and Methods}

\section{Study Design and Patients}

The Institutional Review Board of Taipei Veterans General Hospital approved this descriptive, retrospective chart review study. We screened a consecutive series of 61 ears in 59 children ( $\leq 16$ years) with primary cholesteatomas who underwent tympanomastoidectomy between 1986 and 2010. Among these, 7 surgeries for congenital cholesteatomas were excluded because it is a distinct disease entity from acquired cholesteatoma. Of the remaining 54 potentially eligible ears, $49(91 \%)$ were included in this study, and 5 were excluded due to insufficient data. None of the 49 ears had previous mastoidectomy for chronic otitis media with or without cholesteatoma. All of the operations were performed by C.-F.L., who had already performed $>100$ cholesteatoma surgeries before the start of the research (1986), thereby avoiding the influence of individual surgeons of differing experience on surgical outcomes.

\section{The Cholesteatoma-Atelectasis-Ossicle Staging System of}

\section{Cholesteatoma}

To assist with the evaluation of disease severity, the pre-operative conditions were recorded based on the Cholesteatoma-Atelectasis-Ossicle (CAO) staging system of cholesteatoma (table 1) proposed by Lien [1985]. In this system, 'C' represents the extension of cholesteatoma into the middle ear cleft, 'A' represents the degree of atelectasis according to the Sadé [Sadé and Halevy, 1976] and Tos [Tos and Poulsen, 1980] classifications, and 'O' represents the number of ossicles destroyed by cholesteatoma. Each value of $\mathrm{C}, \mathrm{A}$, and $\mathrm{O}$ is given a score of 1,2 , or 3 based on the degree of severity. The total CAO score thus ranges from 3 to 9 . A score of 3 and 4 represents early-stage cholesteatoma (stage I), 5 and 6 moderate stage (stage II), and 7, 8 and 9 advanced stage (stage III).

\section{Outcome Assessments}

The medical records of all patients were analyzed for the last recidivism-free otologic examinations and outcomes. Data were collected on gender, side of ear, age of surgery, operative findings (i.e. extent of cholesteatoma, degree of atelectasis, and ossicular condition), recidivistic disease (i.e. recurrent and residual cholesteatomas), and pre- and post-operative recidivism-free audiograms. We compiled data on the pre- and post-operative recidivism-free pure-tone average and air-bone gap $(\mathrm{ABG})$, with mean thresholds determined at $0.5,1,2$, and $3 \mathrm{kHz}$. When a threshold at $3 \mathrm{kHz}$ was not available, the average of $2 \mathrm{kHz}$ and $4 \mathrm{kHz}$ was estimated. The ABG was calculated by using air conduction (AC) and bone conduction $(\mathrm{BC})$ thresholds recorded from the same audiogram. The variable used to evaluate a successful hearing outcome was the percentage of ears with $\mathrm{AC} \leq 30 \mathrm{~dB}$ (serviceable hearing).

\section{Surgical Techniques}

The two main approaches for cholesteatoma are canal wall up and canal wall down mastoidectomy [Nikolopoulos and Gerbesiotis, 2009]. However, a technically feasible and safe alternative to conventional canal wall up or down technique, atticotomy-limited mastoidectomy with cartilage reconstruction (inside-out ap- 

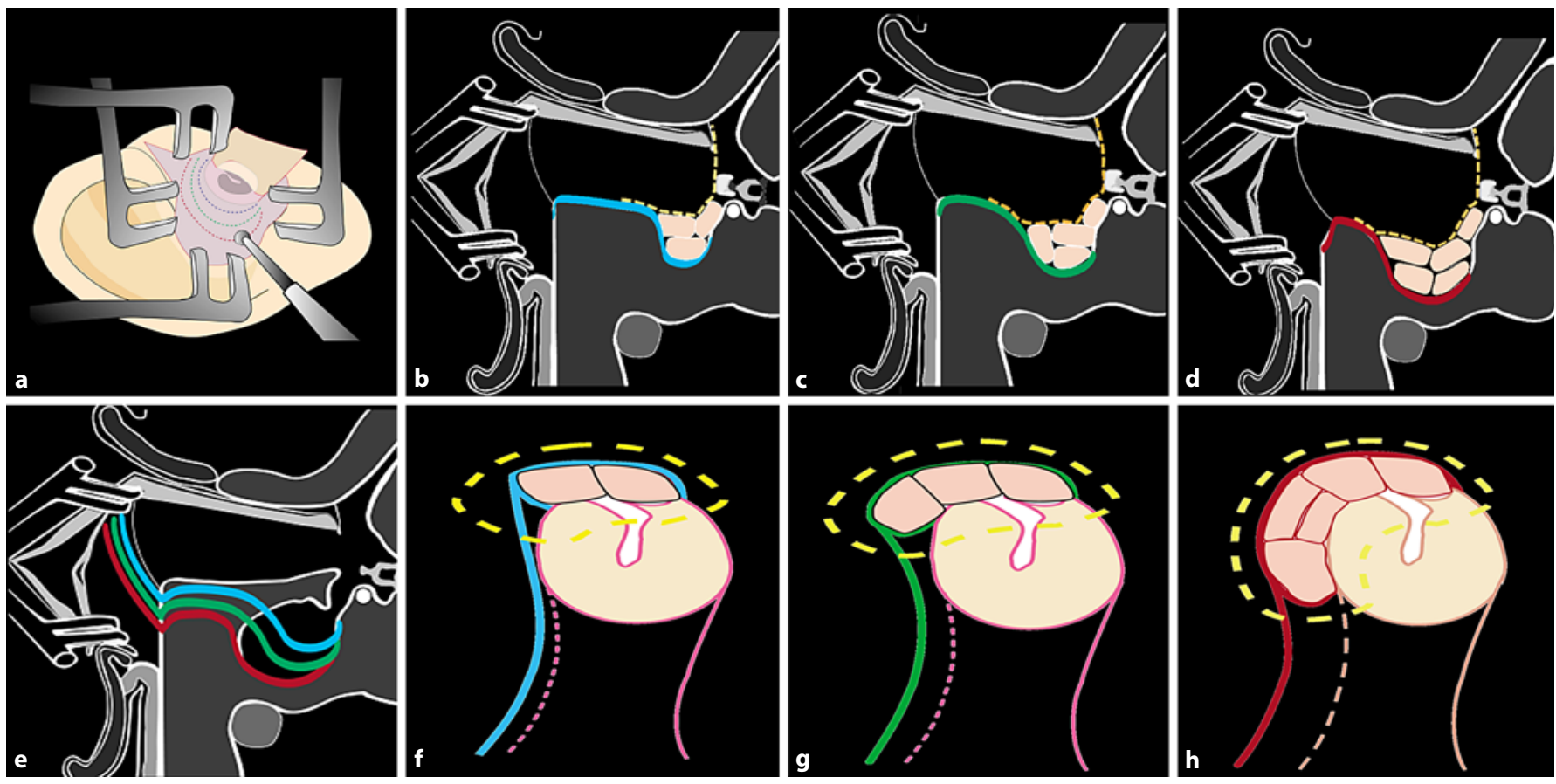

Fig. 1. Atticotomy-limited mastoidectomy with cartilage reconstruction (inside-out approach). An endaural incision is made to expose the EAC (a). Progressive retrograde tympanomastoidectomy is individualized by the extent of the cholesteatoma, thus creating an atticotomy (blue line on $\mathbf{a}, \mathbf{b}, \mathbf{e}, \mathbf{f}$ ), attico-antrectomy

proach), has been widely used by many surgeons in the treatment of cholesteatoma [Dornhoffer, 2000; Lan et al., 2003; DeRowe et al., 2005; Uyar et al., 2006; Nikolopoulos and Gerbesiotis, 2009; Roth and Haeusler, 2009; Hatano et al., 2010]. This method is a less invasive option that produces satisfactory long-term outcomes [Nikolopoulos and Gerbesiotis, 2009]. The senior author (C.-F.L.) has used this method for treating cholesteatoma since 1986. The aim of this modified surgical technique is to achieve less structural destruction and greater preservation of uninvolved middle-ear cleft structures and especially functional mastoid air cells.

This method was performed in a single stage as follows (fig. 1). An endaural incision was made to expose the external auditory canal (EAC) and temporalis fascia (fig. 1a). The fascia was harvested for grafting. After elevating the tympanomeatal flap from the posterior canal wall, the EAC was enlarged and the scutum removed, thus providing direct access to the cholesteatoma. Retrograde mastoidectomy (inside-out approach) was used to remove the cholesteatoma, which extended along the route of disease involvement, thus creating an atticotomy (blue line on fig. 1a, b, e, f), attico-antrectomy (green line on fig. la, c, e, g), or atticoantro-mastoidectomy (red line on fig. 1a, d, e, h) open cavity [Nikolopoulos and Gerbesiotis, 2009]. Cartilage was harvested from either the cavum or cymba conchae or both. The cartilage was cut into pieces, but the perichondrium was preserved, thus allowing fast blood vessel regeneration, early restoration of circulation, and a high survival rate of chondrocytes inside the carti- (green line on $\mathbf{a}, \mathbf{c}, \mathbf{e}, \mathbf{g}$ ), or attico-antro-mastoidectomy (red line on $\mathbf{a}, \mathbf{d}, \mathbf{e}, \mathbf{h}$ ) open cavity. Uninvolved middle ear cleft structures are preserved with mucosa. Cartilage harvested from the choncha is used for cavity obliteration and reconstruction of the posterior canal wall. lage [Tu et al., 2008]. To avoid post-operative cavity problems, the perichondrium-preserved cartilage was obliterated in the 'tailormade' tympanomastoid open cavity for structural support and further aligned to reconstruct the posterior canal wall and maintain the anatomy of the EAC. The alignment and fixation of the cartilage was performed delicately to ensure a smooth surface that had no pockets.

Tympanoplasty was then performed to allow hearing restoration. Myringostapediopexy with interposition grafts using a sculpted incus or malleus head (modified type III tympanoplasty) was performed if the long process of the incus was either necrotic or absent but a mobile footplate and stapes superstructure remained. Myringoplatinopexy with interposition grafts using autogeneic cartilage (modified type IV tympanoplasty) was performed if the stapes superstructure was lost but a mobile footplate remained.

\section{Statistical Analysis}

The paired samples t test was used to calculate significant differences between pre- and post-operative ACs, BCs, and ABGs. Fisher's exact test was used when analyzing categorical variables (the percentage of ears with pre- and post-operative $\mathrm{AC} \leq 30 \mathrm{~dB}$ ). A one-way analysis of variance (ANOVA) followed by Scheffe's post hoc test was used for parametric variables including the evaluation of differences in hearing results between ears assigned as tympanoplasty type I, modified type III and modified type IV. Univariate and multivariate logistic regression analyses were used 
Table 2. Demographic characteristics of the patients

\begin{tabular}{ll}
\hline Variables & \\
\hline Sex & \\
$\quad$ Male & $30(61.2)$ \\
Female & $19(38.8)$ \\
Side of ear & \\
$\quad$ Right & $29(59.2)$ \\
Left & $20(40.8)$ \\
Age, years & $09.49 \pm 3.38(3-16)$ \\
Follow-up period, years & $14.19 \pm 6.39(2-24)$ \\
Stage of CAO system & \\
I & $09(18.4)$ \\
II & $09(18.4)$ \\
III & $31(63.3)$ \\
C score of CAO system & \\
C1 & $11(22.4)$ \\
C2 & $06(12.2)$ \\
C3 & $32(65.3)$ \\
A score of CAO system & \\
A1 & $08(16.3)$ \\
A2 & $13(26.5)$ \\
A3 & $28(57.1)$ \\
O score of CAO system & $15(30.6)$ \\
O1 & $19(38.8)$ \\
O2 & $15(30.6)$ \\
O3 & \\
Tympano I & $10(20.4)$ \\
Modified type III & \\
Modified type IV & \\
\hline
\end{tabular}

Values are means \pm SD with ranges in parentheses or numbers with percentages in parentheses.

to predict the factors that affected long-term hearing success. All statistical analyses were performed using a commercially available software package (SPSS version 18.0, SPSS, Inc., Chicago, Ill., USA). p values of $<0.05$ were considered statistically significant.

\section{Results}

In table 2, we show the demographics of the patients. A total of 49 operations (19 in females and 30 in males) were performed on 47 patients. Two patients experienced bilateral cholesteatomas. The right ear was involved in 29 cases and the left in 20 cases. The mean age at the time of surgery was $9.49 \pm 3.38$ years (range $3-16$ ), and the mean duration of follow-up was $14.19 \pm 6.39$ years (range $2-24)$. Forty-seven (96\%) operations had a follow-up period of $\geq 5$ years, and a $\geq 15$-year follow-up period was
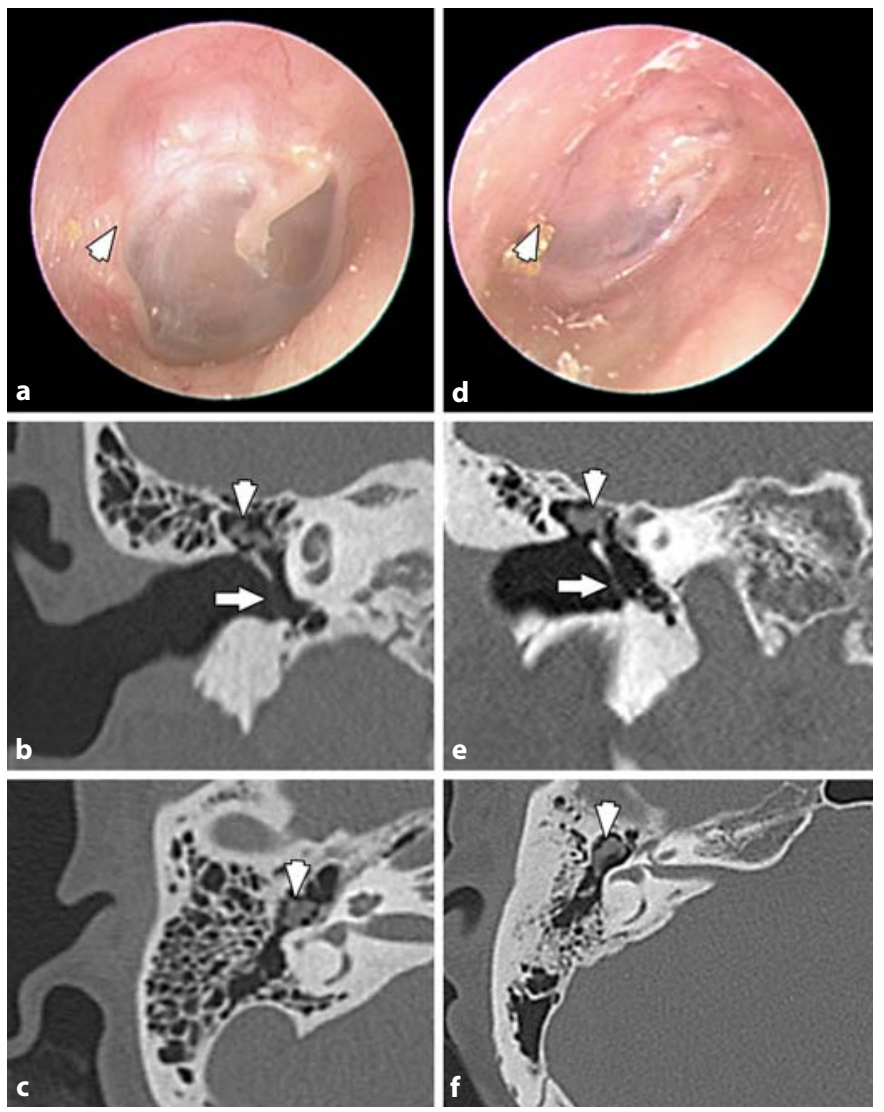

Fig. 2. Attico-antrectomy with cartilage reconstruction (a-c, 11 years of follow-up) and atticotomy with cartilage reconstruction (d-f, 24 years of follow-up). Note the well-maintained cartilage without absorption (arrowhead) and good aeration in the middle ear cleft without eardrum retraction (arrow).

observed in $26(53 \%)$ operations. There were 9,9 , and 31 ears at CAO stage I, II or III, respectively, and 10, 18, and 21 ears underwent type I, modified type III, or modified type IV tympanoplasty, respectively. Analysis of the last recidivism-free otologic examination showed that most patients were capable of maintaining an aerated middleear space with an intact or mild-retracted eardrum (fig. 2). Recidivism of cholesteatoma occurred in 7 ears, including 2 residual and 5 recurrent diseases. The recidivism rate was $14.3 \%$. All of the recidivistic cases underwent revision surgery.

In table 3, we show the pre- and post-operative hearing results. The post-operative ACs $(33.55 \pm 15.42 \mathrm{~dB})$ and ABGs $(17.88 \pm 12.94 \mathrm{~dB})$ were significantly improved when compared with the pre-operative ACs (42.90 \pm $16.47 \mathrm{~dB}, \mathrm{p}<0.001)$ and ABGs $(30.23 \pm 13.68 \mathrm{~dB}, \mathrm{p}<$ 0.001 ); however, there was no significant deterioration of 
Table 3. Comparison of pre- and post-operative audiometric results

\begin{tabular}{lllr}
\hline & Pre-OP, dB & Post-OP, dB & p \\
\hline AC & $42.90 \pm 16.47$ & $33.55 \pm 15.42$ & $<\mathbf{0 . 0 0 1}$ \\
BC & $15.34 \pm 11.09$ & $15.68 \pm 8.27$ & 0.802 \\
ABG & $30.23 \pm 13.68$ & $17.88 \pm 12.94$ & $<\mathbf{0 . 0 0 1}$ \\
AC $<30 \mathrm{~dB}(\mathrm{n}=49)$ & $12(24.5 \%)$ & $20(40.8 \%)$ & $\mathbf{0 . 0 0 8}$ \\
\hline
\end{tabular}

Values are means \pm SD or numbers with percentages in parentheses. Bold values indicate statistical significance. Pre-OP $=$ Pre-operative; Post-OP = post-operative.

Table 4. Pre- and postoperative hearing results for three types of tympanoplasty

\begin{tabular}{|c|c|c|c|c|c|c|c|c|c|c|}
\hline \multirow[t]{2}{*}{ Type } & \multirow{2}{*}{$\begin{array}{l}\text { Ears } \\
(n=49)\end{array}$} & \multicolumn{3}{|c|}{ AC average, $\mathrm{dB}$} & \multicolumn{3}{|c|}{ BC average, $\mathrm{dB}$} & \multicolumn{3}{|c|}{$\mathrm{ABG}$ average, $\mathrm{dB}$} \\
\hline & & pre-OP & post-OP & $\mathrm{p}$ & pre-OP & post-OP & $\mathrm{p}$ & pre-OP & post-OP & $\mathrm{p}$ \\
\hline I & $10(20.4)$ & $30.31 \pm 13.28$ & $22.69 \pm 8.98$ & 0.086 & $14.56 \pm 5.47$ & $12.81 \pm 3.62$ & 0.444 & $18.75 \pm 14.54$ & $09.88 \pm 8.67$ & 0.161 \\
\hline Modified III & $18(36.7)$ & $37.59 \pm 12.97$ & $25.90 \pm 9.68$ & 0.001 & $11.16 \pm 6.07$ & $14.76 \pm 7.96$ & 0.050 & $28.81 \pm 11.06$ & $11.14 \pm 10.25$ & $<0.001$ \\
\hline Modified IV & $21(42.9)$ & $53.44 \pm 14.40$ & $45.29 \pm 14.09$ & 0.028 & $19.29 \pm 14.77$ & $17.83 \pm 9.73$ & 0.835 & $36.92 \pm 11.63$ & $27.46 \pm 10.45$ & $<0.011$ \\
\hline
\end{tabular}

Values are means \pm SD or numbers with percentages in parentheses. Bold values indicate statistical significance. pre-OP $=$ Preoperative; post-OP = post-operative.

Table 5. Predictors of hearing success by univariate and multivariate analyses

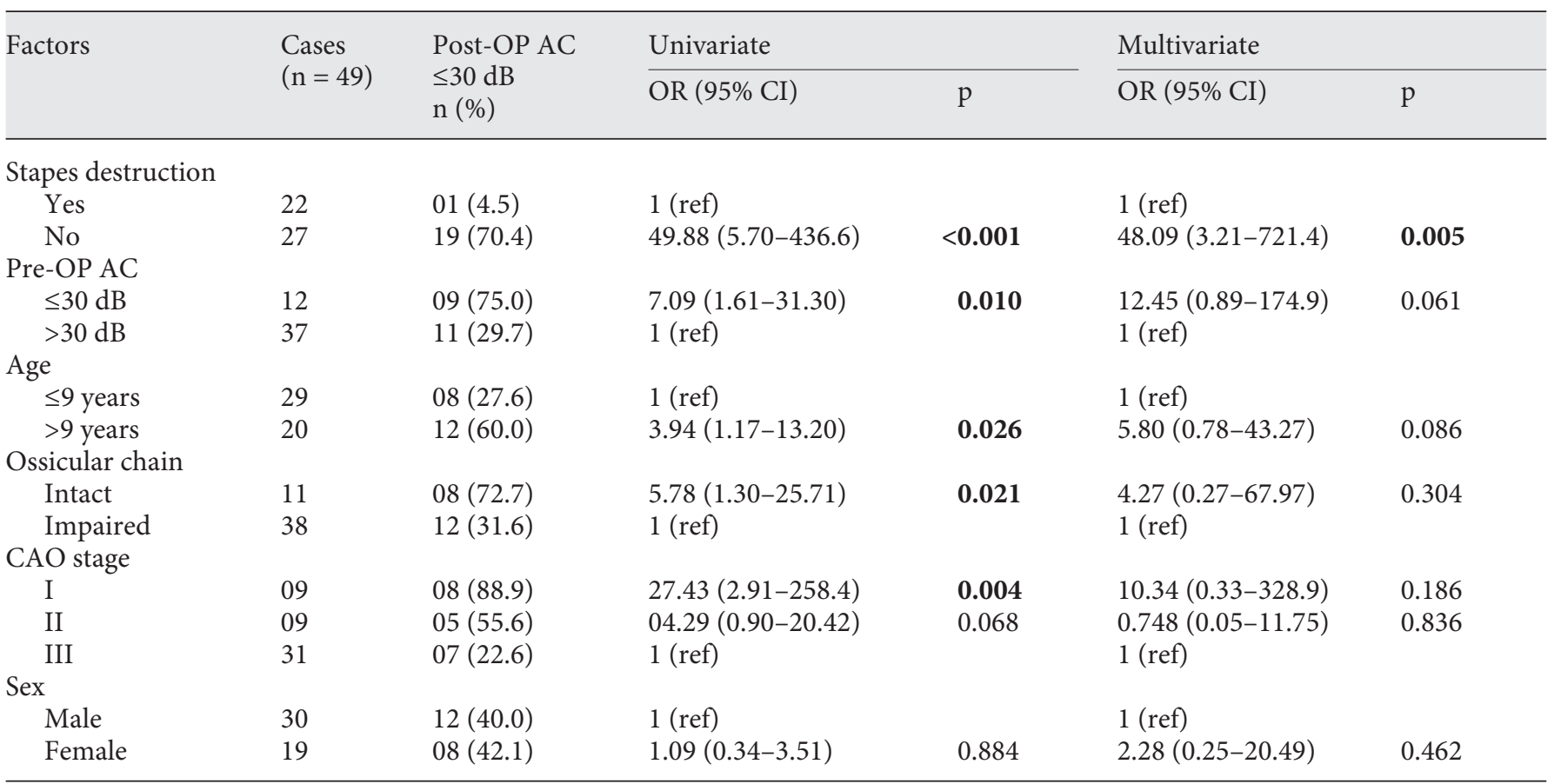

Bold values indicate statistical significance. Pre-OP = Pre-operative; post-OP = post-operative. 
BC post-operatively $(\mathrm{p}=0.802)$. The percentage of ears with AC $\leq 30 \mathrm{~dB}$ after surgery $(40.8 \%)$ was significantly higher than prior to surgery $(24.5 \%, \mathrm{p}=0.008)$.

When comparing pre- and post-operative hearing results of the different tympanoplasties (table 4), the postoperative mean ACs for ears with modified type III (25.90 $\pm 9.68 \mathrm{~dB})$ and modified type IV (45.29 $\pm 14.09 \mathrm{~dB})$ tympanoplasties were significantly smaller than the preoperative mean ACs $(37.59 \pm 12.97 \mathrm{~dB}$ for modified type III, $\mathrm{p}=0.001$, and $53.44 \pm 14.40 \mathrm{~dB}$ for modified type IV, $\mathrm{p}=0.028)$. Similarly, the post-operative mean ABGs for ears with modified type III $(11.14 \pm 10.25 \mathrm{~dB})$ and modified type IV (27.46 $\pm 10.45 \mathrm{~dB})$ tympanoplasties were also significantly smaller than the pre-operative mean ABGs (28.81 $\pm 11.06 \mathrm{~dB}$ for modified type III, $\mathrm{p}<0.001$, and $36.92 \pm 11.63 \mathrm{~dB}$ for modified type IV, $\mathrm{p}=0.011$ ). Although the post-operative mean ACs and ABGs for ears with type I tympanoplasty were smaller than the pre-operative mean ACs and ABGs, the difference was not statistically significant $(\mathrm{p}=0.086$ and 0.161 , respectively). There was no significant deterioration in BC postoperatively for ears with tympanoplasty type I, modified type III, or modified type IV ( $\mathrm{p}=0.444,0.050$, and 0.835 , respectively).

A one-way ANOVA revealed a statistically significant difference in post-operative mean $\mathrm{AC}$ (d.f. $=2 ; \mathrm{F}=18.76$; $\mathrm{p}<0.001)$ and $A B G$ (d.f. $=2 ; F=16.76 ; p<0.001$ ) for the three tympanoplasties. Moreover, Scheffe's post hoc test indicated that the post-operative mean ACs and ABGs for ears with modified type IV tympanoplasty were worse than those for the ears with type I or modified type III tympanoplasties $(\mathrm{p}<0.001)$.

In table 5, we show the logistic regression analyses used to predict the factors that affect long-term hearing success. In a univariate analysis of the predictors, the probability of hearing success was significantly affected by intact stapes ( $\mathrm{p}<0.001$; odds ratio, OR $=49.88$; $95 \%$ confidence interval, CI 5.70-436.6), pre-operative AC $\leq 30 \mathrm{~dB}(\mathrm{p}=0.010$; OR $=7.09$; 95\% CI 1.61-31.30), older age ( $>9$ years, $\mathrm{p}=0.026 ; \mathrm{OR}=3.94 ; 95 \% \mathrm{CI} 1.17-13.20)$, ossicular chain integrity $(\mathrm{p}=0.021$; OR $=5.78 ; 95 \% \mathrm{CI}$ $1.30-25.71)$, and early-stage disease $(\mathrm{p}=0.004$; OR $=$ 27.43; 95\% CI 2.91-258.4). Hearing success was not predicted by gender $(\mathrm{p}=0.884)$. Multivariate logistic regression analysis revealed a statistically significant correlation between hearing success and intact stapes only $(\mathrm{p}=$ 0.005 ; OR $=48.09 ; 95 \%$ CI 3.21-721.4). No other factor included into the analysis (i.e. pre-operative $\mathrm{AC} \leq 30 \mathrm{~dB}$, age, ossicular chain integrity, disease severity, and gender) significantly affected hearing success $(p>0.05)$.

\section{Discussion}

We observed that the post-operative mean ACs and ABGs were significantly improved compared with the pre-operative mean ACs and ABGs. In addition, the probability of hearing success was also significantly higher than prior to surgery. The post-operative hearing improvement in our study is consistent with other researchers investigating hearing outcomes in children following cholesteatoma surgery [Murphy and Wallis, 1998; Iino et al., 2001; Chadha et al., 2006; Sun et al., 2010], although we report longer-term hearing results with a mean follow-up of 14 years.

With regard to the different tympanoplasties, significant improvements in ACs and ABGs were not observed post-operatively in type I tympanoplasty; however, significant improvements were observed in modified type III and modified type IV tympanoplasties. This finding may be related to the better pre-operative hearing level in type I tympanoplasty. A one-way ANOVA followed by Scheffe's post hoc test indicated that the ears with modified type IV tympanoplasty had the worst post-operative hearing outcomes with respect to the mean AC and ABG. This finding may be related to the worse pre-operative hearing level in modified type IV tympanoplasty.

Hearing impairment in children, if not managed in time, may delay language development and cause difficulty in comprehension, which may hinder progress at school. Children with impaired hearing are often socially isolated [Most, 2004]. Even children with unilateral hearing loss may experience educational and/or behavioral complications. Of children with unilateral loss, Bess et al. [1986] reported that $33 \%$ failed in school and had to repeat a class and $40 \%$ exhibited behavioral difficulties. The costs for consultation, investigation, and treatment due to hearing impairment can also impose a heavy socio-economic burden on families and countries.

Despite the importance of hearing for children, complete eradication of cholesteatoma rather than hearing preservation is often considered the primary surgical goal for surgeons. Unfortunately, this goal results in conflicts between surgeons and parents because the parents, in contrast, measure the success of the operation based on the correction of hearing impairment [Hamilton, 2010]. Although parents realize that complete disease eradication is important for children, they are more concerned with preservation of hearing in their child after the surgery, in particular if hearing levels are within normal limits before the operation. Therefore, surgeons should pay equal attention to both hearing preservation 
and disease eradication and attempt to achieve both goals.

This study emphasized long-term rather than shortterm observations on hearing changes in children following cholesteatoma surgery because the post-operative course may be affected by various undesirable factors, thus making long-term hearing outcomes unpredictable. First, the natural course of the disease is more aggressive, and it reoccurs more frequently, in children than adults. Second, children are much more prone to recurrent upper respiratory tract infections. The eustachian tube in children is shorter, which allows infectious organisms to enter more easily, and it is at a more horizontal angle, which leads to inadequate drainage of the middle ear. Moreover, the development of the mastoid air cell system is initiated at birth and develops until the age of 10-15 years [Rubensohn, 1965]. The constant change in the size of the mastoid air cells during childhood may result in post-operative instability of the middle ear cleft. Mishiro et al. [2010] found that the outcomes following ossiculoplasty deteriorated significantly during long-term followup, in particular in ears with cholesteatoma. Taken together, long-term follow-up is clearly necessary to identify the functional hearing results and to evaluate the entire outcome context of surgical techniques in pediatric cholesteatoma surgery.

This study verified that long-term hearing preservation is attainable in pediatric cholesteatoma surgery using atticotomy-limited mastoidectomy with cartilage reconstruction (inside-out approach). C.-F.L. has used this method for treating pediatric cholesteatoma since 1986 based on the following considerations. First, as a tertiary medical center in Taiwan, many of our patients come from rural areas of the country, and the need for repeated cleaning of the open cavity is problematic for children and parents. By using cartilage for attic or posterior canal wall reconstruction, the cavity issues can be avoided. Second, there has been a trend towards the use of less radical surgery with functional preservation in many fields. For example, functional endoscopic sinus surgery has been applied for the treatment of chronic rhinosinusitis, and transoral laser microsurgery has been used for laryngeal and hypopharyngeal cancers to avoid total laryngectomy. In pediatric cholesteatoma surgery, many surgeons are less likely to utilize 'radical' wall-down approaches due to the prospect of life-long mastoid cavity maintenance [Preciado, 2012]. Hence, the concept of surgeries that preserve function has aroused the interest of some otologists for clinical application for cholesteatoma [Dornhoffer, 2000; Lan et al., 2003; DeRowe et al., 2005; Uyar et al.,
2006; Nikolopoulos and Gerbesiotis, 2009; Roth and Haeusler, 2009; Hatano et al., 2010]. The goal of this method is to create less structural damage and to maintain greater preservation of the uninvolved functional middle ear cleft. Furthermore, functional cholesteatoma surgery with tympanoplasty allows for satisfactory hearing results, and a revision surgery is rarely required, making this functional technique an attractive alternative to the standard canal wall up and canal wall down procedures [Nikolopoulos and Gerbesiotis, 2009].

The possible reasons underlying hearing preservation in children following cholesteatoma surgery cannot be readily explained by any single mechanism. One such reason may be that an association with sensorineural hearing loss is rarely observed in pediatric populations, even in those with cholesteatoma [Iino et al., 2001]. As noted in our study, there was no significant deterioration in BC over a mean follow-up of 14 years. Additionally, based on our long-term clinical observations in this study, we propose another possible physiological mechanism that has the potential to contribute to hearing preservation in children following cholesteatoma surgery. The key to this physiological mechanism is the mastoid pneumatic system. It is a functional organ and primarily acts as a buffer to pressure changes in the middle ear. This buffer effect prevents eardrum retraction, which has been thought to be associated with mucosal adhesion to the ossicles and subsequent hearing impairment [Borgstein et al., 2008]. Consequently, preserving the uninvolved mucosa in the antrum and mastoid leads to recovery of aeration and ciliary function in the mastoid during the post-operative period [Dornhoffer, 2000]. This method thus prevents post-operative eardrum retraction (fig. 2), effectively preserving a child's long-term hearing. The concept of a pressure-buffering mechanism was further supported by Sadé [1997], who observed that retraction of the pars flaccida almost always occurs in non-pneumatized ears and not in well-pneumatized ears. Furthermore, Merchant et al. [1997] stated that a well-aerated environment of the middle ear enables the eardrum and ossicles to move efficiently, thus guaranteeing good sound transmission.

Knowledge of the factors that are predictive of hearing success would be very useful during surgery and for postoperative observation. Univariate and multivariate logistic regression analyses were performed to assess the correlation between clinical factors and hearing success. However, only stapes integrity was shown to be an independent predictor of hearing success in both univariate and multivariate analyses. It is worth noting that a uni- 
variate analysis showed that pre-operative serviceable hearing ( $\mathrm{AC} \leq 30 \mathrm{~dB}$ ) was a significant favorable predictor $(\mathrm{p}=0.010)$ and that a multivariate analysis showed it as a borderline significant predictor $(\mathrm{p}=0.061)$. This finding is supported by Chadha et al. [2006], who, using a multiple linear regression, reported that $\mathrm{AC}$ threshold was increased by pre-operative AC threshold. The association between pre- and post-operative serviceable hearing provides parents with valuable pre-operative information because they are more concerned with preserving their child's hearing after surgery, particularly if the preoperative hearing is serviceable. In this study, we used 9 years as the cutoff for age at surgery because the mean age of the children in this study was 9.49 years. Although it is likely that surgical stress and repeated post-operative local treatment may be more tolerated by older children, an older age does not guarantee better long-term hearing preservation for children. Our finding is consistent with those of Iino et al. [2001] and Chadha et al. [2006]. They found no correlation between hearing improvement and age. In addition, it may be reasonable to expect that ossicular chain integrity predicts better hearing results. $\mathrm{Cu}-$ riously, however, our findings do not support this expectation. Similarly, we did not find an association between disease severity and hearing success. Nevertheless, modified type IV tympanoplasty and stapes destruction, which may both be used as proxies for disease severity, were found to be associated with poorer outcomes.

Importantly, the definition of post-operative hearing success may vary between otologists and parents. In this study, although there was a statistically significant increase in serviceable hearing from $24.5 \%$ pre-operatively to $40.8 \%$ post-operatively, most families would not consider this $41 \%$ chance of serviceable hearing great odds. However, functional cholesteatoma surgery with hearing reconstruction is nevertheless recommended. Although the gain in hearing is not satisfactory and is insufficient to cure the children, it may stop or delay disease progression. Moreover, it provides for better hearing aid use due to the important discriminatory improvement. Children can thus be expected to use hearing aids more successfully compared with those without hearing reconstructive surgery. However, it must be emphasized that even with excellent technique, it can be quite difficult to achieve excellent hearing results following cholesteatoma surgery. This finding, along with the fact that without disease eradication the patient remains at risk from disease, may be one of the reasons why surgeons appear to cite complete eradication of cholesteatoma rather than hearing preservation as the primary goal of the surgery.
Some possible limitations should be discussed. First, this study was limited by the small number of patients. Second, although the uniformity and consistency offered by a single surgeon/single center performing the cholesteatoma surgeries is a great strength of our study because it reduces the influence of individual surgeons on surgical outcomes, our results may not be directly applicable to other clinics and surgeons because other surgeons may use different techniques and because surgical skills may differ among surgeons. Furthermore, the limitations of this study also include the inherent bias associated with a retrospective review and the absence of a control group for these outcome observations. Moreover, although the concept of functional microsurgery (i.e. retrograde atticotomy-limited mastoidectomy) for cholesteatoma has been used widely [Dornhoffer, 2000; Lan et al., 2003; DeRowe et al., 2005; Uyar et al., 2006; Nikolopoulos and Gerbesiotis, 2009; Roth and Haeusler, 2009; Hatano et al., 2010], otologists may have made slightly different modifications to this technique. Consequently, our results may not be easily extrapolated to other population groups that have been operated on using different modified methods. With regard to the prediction model in this study, other predictor variables that have equal or greater influence on hearing outcomes may not be included in the logistic regression analyses. In addition, a statistically significant correlation between the prognostic factors and outcome variables does not necessarily mean that a causal relation exists. Therefore, the results of the regression analysis must be interpreted with caution.

\section{Conclusions}

Effective long-term hearing preservation is attainable in children following atticotomy-limited mastoidectomy with cartilage reconstruction (inside-out approach). Our research may offer ways to overcome some of the limitations concerning hearing preservation following cholesteatoma surgery. In addition, we found that stapes destruction was an independent negative prognostic determinant of achieving hearing success, and it may provide patients and parents with information on the expected hearing outcome.

\section{Disclosure Statement}

All authors declare that they have no conflicts of interest. 


\section{References}

-Bess FH, Klee T, Culbertson JL: Identification, assessment, and management of children with unilateral sensorineural hearing loss. Ear Hear 1986;7:43-51.

Borgstein J, Gerritsma TV, Bruce IA: Erosion of the incus in pediatric posterior tympanic membrane retraction pockets without cholesteatoma. Int J Pediatr Otorhinolaryngol 2008;72:1419-1423.

- Chadha NK, Jardine A, Owens D, Gillett S, Robinson PJ, Maw AR: A multivariate analysis of the factors predicting hearing outcome after surgery for cholesteatoma in children. J Laryngol Otol 2006;120:908-913.

-DeRowe A, Stein G, Fishman G, Berco E, Avraham S, Landsberg R, Sadé J: Long-term outcome of atticotomy for cholesteatoma in children. Otol Neurotol 2005;26:472-475.

Dornhoffer JL: Retrograde mastoidectomy with canal wall reconstruction: a single-stage technique for cholesteatoma removal. Ann Otol Rhinol Laryngol 2000;109:1033-1039.

Hamilton JW: Systematic preservation of the ossicular chain in cholesteatoma surgery using a fiber-guided laser. Otol Neurotol 2010;31: 1104-1108.

-Hatano M, Ito M, Yoshizaki T: Retrograde mastoidectomy on demand with soft-wall reconstruction in pediatric cholesteatoma. Acta Otolaryngol 2010;130:1113-1118.

- Iino Y, Nagamine H, Sasaki Y, Kodera K: Hearing results of canal wall reconstruction tympanoplasty for middle ear cholesteatoma in children. Int J Pediatr Otorhinolaryngol 2001;60:65-72.
Lan MY, Lien CF, Liao WH: Using high resolution computed tomography to evaluate middle ear cleft aeration of postoperative cholesteatoma ears. J Chin Med Assoc 2003;66: 217-223.

Lien CF: Staging of Cholesteatoma. Amsterdam, Elsevier Science Publishers BV, 1985.

- Merchant SN, Ravicz ME, Puria S, Voss SE, Whittemore KR Jr, Peake WT, Rosowski JJ: Analysis of middle ear mechanics and application to diseased and reconstructed ears. Am J Otol 1997;18:139-154.

Mishiro Y, Sakagami M, Kitahara T, Kakutani C: Prognostic factors of long-term outcomes af ter ossiculoplasty using multivariate analysis. Eur Arch Otorhinolaryngol 2010;267: 861-865.

Most T: The effects of degree and type of hearing loss on children's performance in class. Deaf Education Int 2004;6:154-166.

Murphy TP, Wallis DL: Hearing results in pediatric patients after canal-wall-up and canalwall-down mastoid surgery. Otolaryngol Head Neck Surg 1998;119:439-443.

Nikolopoulos TP, Gerbesiotis P: Surgical management of cholesteatoma: the two main options and the third way - atticotomy/limited mastoidectomy. Int J Pediatr Otorhinolaryngol 2009;73:1222-1227.

Preciado DA: Biology of cholesteatoma: special considerations in pediatric patients. Int $\mathrm{J} \mathrm{Pe}-$ diatr Otorhinolaryngol 2012;76:319-321.
Roth TN, Haeusler R: Inside-out technique cholesteatoma surgery: a retrospective longterm analysis of 604 operated ears between 1992 and 2006. Otol Neurotol 2009;30:5963.

Rubensohn G: Mastoid pneumatization in children at various ages. Acta Otolaryngol 1965; 60:11-14.

Sadé J: On the function of the pars flaccida: retraction of the pars flaccida and buffering of negative middle ear pressure. Acta Otolaryngol 1997;117:289-292.

Sadé J, Halevy A: The natural history of chronic otitis media. J Laryngol Otol 1976;90:743751.

- Sun J, Hu Y, Lv Q, Wang Y, Li X, Xu W, Wang S Mei Z, Wan G: Canal wall-down mastoidectomy with mastoid obliteration for pediatric cholesteatoma. Acta Otolaryngol 2010;130: 259-262.

Tos M, Poulsen G: Attic retractions following secretory otitis. Acta Otolaryngol 1980;89: 479-486.

Tu TY, Yang AH, Fan CC: Short-term effects of perichondrium preservation on chondrocyte survival and chondrogenesis of auricular cartilage grafted in rabbit tympanic bullae. Audiol Neurootol 2008;13:239-246.

-Uyar Y, Ozturk K, Keles B, Arbag H, Ulku CH: Anterior atticoantrostomy for cholesteatoma surgery. Ann Otol Rhinol Laryngol 2006; 115:150-155. 